\title{
Calibrated modelling of form-active structures
}

\author{
Stephen Melville ${ }^{1}$ Cecilie Brandt-Olsen ${ }^{1}$, John Harding ${ }^{2}$ \\ 1. Format Engineers, Bath, UK. \\ 2. University of The West of England, UK
}

\begin{abstract}
The design and analysis of a bending active, thin timber shell structure using a self-written physics based structural analysis solver, K2Engineering.
\end{abstract}

\section{Keywords}

Active bending, timber gridshells, structural morphology, design exploration, geodesic curves, doubly curved shells, real-time analysis.

\section{Introduction}

Lightweight and low stiffness structures which exploit an efficiency of form to resist applied loads in mainly direct tension or compression with minimal bending of the members are often difficult to model and to analyse. Gridshell structures are an example of these, which whilst elegant in form are often limited in terms of design exploration by the need to incorporate a form finding step which is dictated by the force flow. Their slender nature can mean that the assumption of a linear relationship between force and deformation no longer applies due to the inherently large deformations and global/local buckling behaviour.

The steps to address both the form finding as well as the large deformations and buckling should, ideally, be highly interconnected. It would also be advantageous for any design workflow to allow an openness that allows for intervention in geometry and topography of structure in a simple and instantaneous way. Conventional structural analysis software does not offer these steps in a single platform. They are only useful for form-finding or the analysis is so cumbersome to set up that multiple design iterations become impractical.

In an attempt to improve this workflow, a plug-in called "K2Engineering" has been developed for the parametric modelling environment Grasshopper. K2Engineering offers the ability to analyse form-active structures in a much more interactive environment and thereby encourages an informed exploration in the early design stage. It is an extension of the physics constraint solver "Kangaroo2" developed by Daniel Piker. The physics based underpinnings of the plug-in allow swift evaluation of bending active structures and accurate modelling of the resulting geometry caused by applied bending forces.

This paper focuses on a doubly curved gridshell using bending-active thin plywood timber laths, designed and erected by students at The University of The West of England, the structural performance of which was simulated in K2Engineering. 


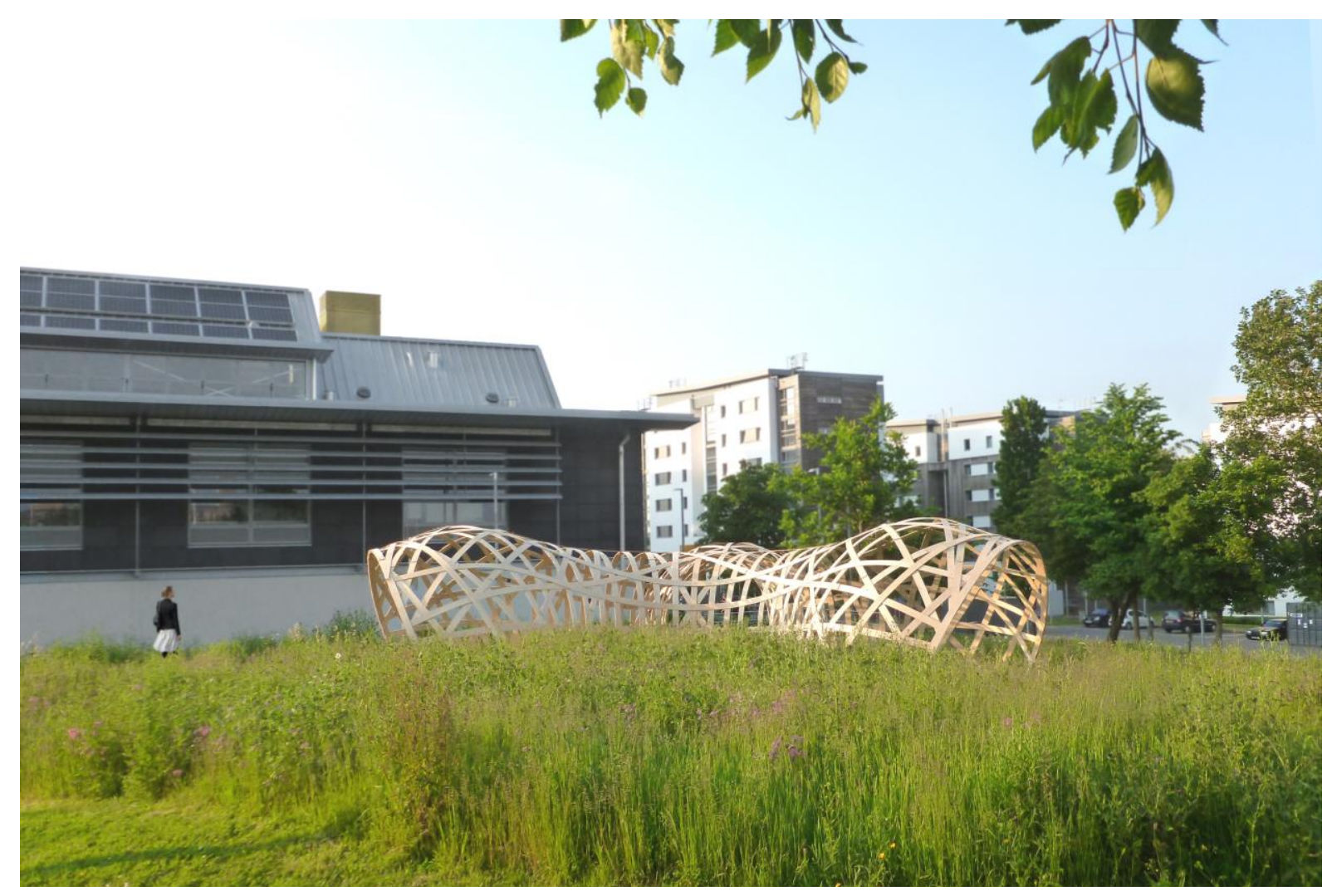

Figure 1. The completed pavilion

\subsection{Geometry modelling}

A doubly curved surface to suit the site constraints was defined parametrically (using Rhino/Grasshopper) and then used to derive a set of primary laths based on geodesic lines. Secondary elements were then woven around these primaries, again by generating geodesics. Finally, splice points along the laths were located, with the final geometry the exported for detailed design. The use of $6.5 \mathrm{~mm}$ thick birch plywood was essential to achieve the bending radii whilst still retaining the required shell strength at such a thin depth. Similar methods of assembly were previously explored on the Ongreening Pavilion [1] with flat timber laths, albeit within a controlled internal environment.

\section{Analysis methodology}

Early in the structural analysis process it was evident that a conventional finite element solver was unable to accurately predict non-linear buckling and was also unable to take into account the pre-stress of the bent laths. We therefore made use of the K2Engineering components to simulate the non-linear behaviour of the structure and to investigate its post buckling response.

The pre-stress from the bent laths was included to establish how that affected the initial deformation of the gridshell firstly under its own weight and then applied external loads. Next was a study of the non-linear buckling analysis using dynamic relaxation to find equilibrium for each load increment whilst tracing the displacement and observing any sudden "jumps". We concluded that the shell had a very ductile behaviour but with a sufficiently high buckling load 
factor to give confidence that the structure would not fail. The buckling analysis was in part based on previous research by the author [2].

The stresses were validated using the forces from finite element analysis (another plug-in for the Grasshopper eco-system, Karamba) with superimposed stresses from the initial curvature. K2Engineering is currently limited to symmetrical cross sections so care had to be taken to accurately simulate the rectangular cross sections of the laths; hence a weighted average of the moment of inertia in both directions was used until similar deflections between the Karamba finite element model and the K2Engineering model could be seen.

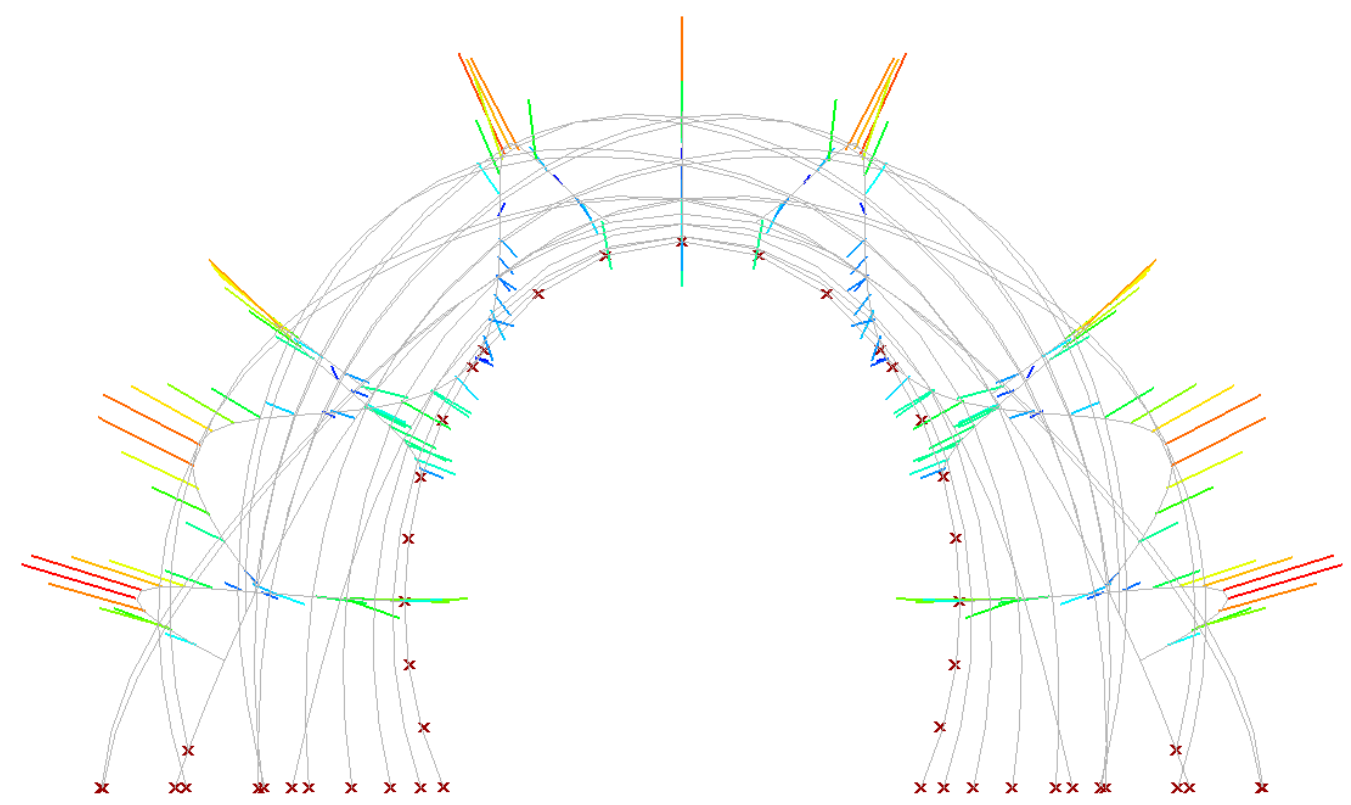

Figure 2. Stress analysis at the pavilion entrance using K2 Engineering

\section{Conclusions}

The built form and its real life behaviour corresponded well to the predictions made by the software. The pavilion shows what can be achieved with computational design techniques when using traditional low cost and low-carbon material such as timber and relatively simple fabrication techniques as a constraint. The K2Enginering plug-in was able to simulate the geometry created by bending forces and to predict the non-linear buckling behaviour of the structure and as such is a useful tool in an interactive design process.

\section{References}

[1] Harding $\mathrm{J}$ and Pearson W and Lewis $\mathrm{H}$ and Melville S. The Ongreening Pavilion. Advances in Architectural Geometry, 2014; 295-308.

[2] Brandt-Olsen C. Harmonic Form-Finding for the design of curvature stiffened shells. MPhil thesis, Technical University of Denmark. 